\title{
Automatic Detection of Myocardial Infarction Through a Global Shape Feature Based on Local Statistical Modeling
}

\author{
Mahdi Tabassian ${ }^{1,2}$, Martino Alessandrini ${ }^{2}$, Peter Claes $^{3}$, Luca De Marchi ${ }^{1}$, \\ Dirk Vandermeulen ${ }^{3}$, Guido Masetti ${ }^{1}$ and Jan D'hooge ${ }^{2}$ \\ 1 Dep. of Electrical, Electronic and Information Engineering, \\ University of Bologna, Bologna, Italy \\ mahdi.tabassian2@unibo.it \\ ${ }^{2}$ Dep. of Cardiovascular Sciences, Cardiovascular Imaging and Dynamics Group, \\ KU Leuven, Leuven, Belgium \\ 3 Dep. of Electrical Engineering-ESAT, Medical Imaging Research Center (MIRC), \\ KU Leuven, Leuven, Belgium
}

\begin{abstract}
This paper presents a local-to-global statistical approach for modeling the major components of left ventricular (LV) shape using its 3 -D landmark representation. The rationale for dividing the LV into local areas is bi-fold: 1) to better identify abnormalities that lead to local shape remodeling and, 2) to decrease the number of shape variables by using a limited set of landmark points for an efficient statistical parametrization. Principal Component Analysis (PCA) is used for the statistical modeling of the local regions and subsets of the learned parameters that provide significant discriminatory information are taken from each local model in a feature selection stage. The selected local parameters are then concatenated to form a global representation of the LV and to train a classifier for differentiating between normal and infarcted LV shapes.
\end{abstract}

Keywords: Local statistical shape modeling, Principal Component Analysis, feature selection, myocardial abnormality detection

\section{Introduction}

Statistical shape analysis is a promising approach to model cardiac anatomy and to characterize myocardial abnormalities. The success of the point distribution model (PDM) [2] in describing anatomical structures of medical images makes it the basis of the majority of cardiac shape parametrization algorithms. These algorithms have been established using both linear methods (such as Principal Component Analysis (PCA) [7, 12, 14, 18] and Independent Component Analysis (ICA) [15]) and nonlinear techniques (such as kernel PCA [5]). One drawback of these techniques, however, is that they treat the shape globally. In addition to being computationally expensive due to the requirement of modeling large number of variables, a global approach may fail to characterize abnormalities that affect small regions of the myocardium. An alternative approach is to learn 
local statistical shape components and then merge their results to describe the global shape as a poly-local model. A recent and well-established example of such framework is presented in [17]. It is based on utilizing local shape descriptors, but not landmark points as suggested in PDM, and employing a manifold learning technique called ISOMAP [16] for dimension reduction.

Inspired by [17], a local statistical shape modeling approach based on PDM and PCA [9] is presented in this paper to characterize major components of LV shapes. The rationale of utilizing PCA in our framework is as following: 1) PCA implementation involves simple steps and its parameters can be efficiently computed and, 2) it allows to visualize major modes of data variation. The latter property could be of particular interest to study the relation between the parameters of the statistical model and patho-physiology of the heart.

The main contribution of this paper compared to the framework presented in [17] is the way that the local statistical information are incorporated in the classification phase. In [17], an independent classifier was built with the parameters of each local model and the classifiers' decisions were fused with the majority voting rule. Independent treatment of the local models' parameters could degrade the capability of the combined classification model in dealing with abnormalities that affect small regions of the heart. Here, we propose to create an alternative local-to-global representation of the LV shape components by concatenating the parameters of the local models and then building a classifier with the obtained feature vector. Having the advantage of encoding global shape parameters of the $\mathrm{LV}$, spatial relation between the local zones is taken into consideration by using the feature concatenation technique. Explicit usage of the local statistical parameters can also create distinct areas in the global feature space. This property enables a classification system to better characterize abnormalities that mostly affect small regions of the myocardium.

\section{Materials and Methods}

Fig. 1 represents global and local architectures that were implemented in this paper for the statistical modeling and classification of LV shapes. In the local architecture (Fig. 1(b)), the LV was divided into non-overlapping regions of interest (ROI) and an independent PCA model was built with the local shapes belonging to each ROI. By taking a subset of the learned statistical parameters, two different classification schemes were examined. In addition, a global PCA model (Fig. 1(a)) was also built to benchmark the performances of the local PCA models.

\subsection{Data and Preprocessing}

A data set of 100 healthy volunteers and 100 patients with myocardial infarction from the MESA [1] and DETERMINE studies [10] respectively, was used in our experiments. These data sets are part of the Cardiac Atlas Project (CAP, www.cardiacatlas.org) [6] and contain cardiovascular magnetic resonance (CMR) 


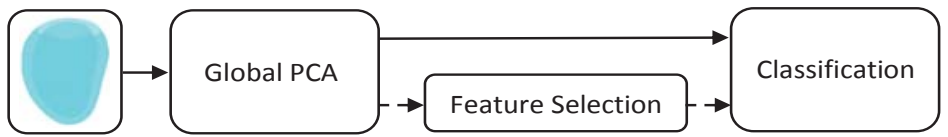

(a)

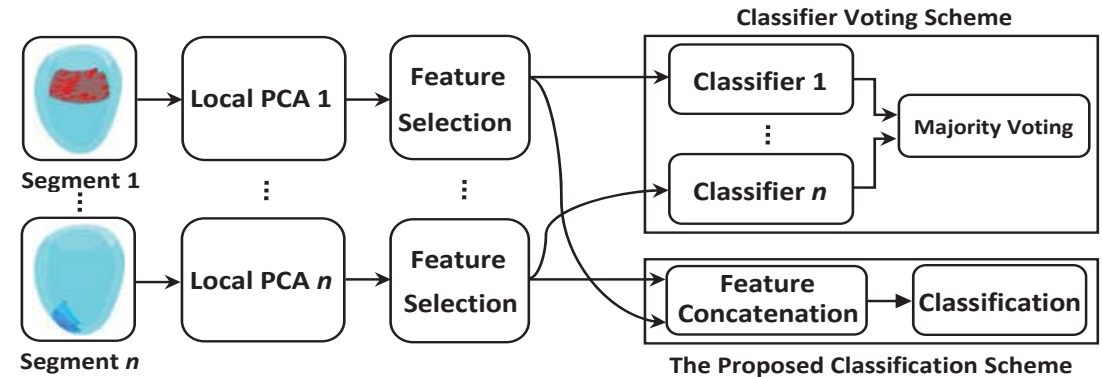

(b)

Fig. 1. Architectures of the implemented statistical frameworks. (a) A PCA model is constructed with whole LV shapes. Then, a subset of the first PCs (solid line) or selected PCs (dashed line) is used for training a classifier. (b) Independent PCA models are built with the segments of LV shapes and subsets of the selected PCs are used in the classification phase. In the classifier voting scheme, independent classifiers are trained with the selected PCs of the local models and the final decision is made by the majority voting rule. In the proposed scheme, one classifier is trained with a feature vector that is obtained by concatenating the local models' parameters.

images. Endocardial and epicardial shapes at end-diastole (ED) and end-systole (ES) are represented with their corresponding Cartesian sets of landmark points in magnet coordinates. It has been demonstrated in [18] that a PCA model built with shape vectors at ED and ES could provide better outcomes than its counterparts that were constructed with only ED or ES shapes. Therefore, the shapes of both ED and ES cardiac phases were used for the implementation of the global and local PCA models in the current study.

All shapes were aligned by making use of the generalized Procrustes superimposition method [13]. As suggested in [18], for building the global PCA model the alignment phase has been performed by eliminating position and orientation differences but preserving scale variations as ventricular size has a predictive value for diagnosing myocardial infarction. For constructing the local PCA models, however, scale variations were also removed in the alignment procedure.

\subsection{Statistical Modeling}

Both global and local PCA models were learned using the data of the healthy volunteers to capture major modes of normal shape variations. For building the local PCA models, small, medium and large ROI sizes were examined which respectively encompassed 4,8 and 16 faces of the 3-D meshes in both the cir- 
cumferential and longitudinal directions where the full LV mesh was composed of $32 \times 32$ faces. Note that the landmark points in each ROI were consistent across the subjects.

\subsection{Feature Selection}

Traditionally, data are projected onto the subspace spanned by the first principal components (PCs) to retain most of the variation in the original variables. However, it is possible that some of the PCs with low contribution in the data variation contain relevant discriminatory information. As such, the $P$-metric method [8] was used in our framework to select relevant PCs:

$$
P\left(P C_{i}\right)=\frac{\left|\mu_{1}-\mu_{2}\right|}{\sigma_{1}+\sigma_{2}}
$$

where $\mu_{1}$ and $\mu_{2}$ are respectively the means of the normal and infarcted samples after projecting onto the subspace of the $i$ th $\mathrm{PC}$ and $\sigma_{1}$ and $\sigma_{2}$ are their corresponding standard deviations. A high $P$-metric value for a $\mathrm{PC}$ implies that it provides a good separation between the samples of the two classes. Therefore, the PCs were sorted based on their $P$-metric values in descending order and a subset of the first selected PCs were used in the classification stage.

\subsection{Classification}

Fig. 1(b) illustrates two classification schemes that were trained with the parameters of the local PCA models. The first scheme uses the strategy proposed in [17] while the second one works based on the idea of concatenating the local PCA models' parameters. Both methodologies were implemented by making use of a subset of PCs that have been chosen in the feature selection stage. Note that, since the local PCA models were trained independently, the selected PCs for each model can be different from the others. SVM [3] with a linear kernel was used as classifier in both global and local models. Classification outcomes were achieved using 10-fold cross-validation.

\section{Results}

Average classification outcomes obtained with the global PCA model are shown in Fig. 2. While the best result of the classifier trained with the first PCs (Fig. $2(\mathrm{a})$ ) was achieved by preserving $99 \%$ of the data variation (the first $60 \mathrm{PCs}$ ), training the classifier with the selected PCs (Fig. 2(b)) yielded better performance with considerably less number of features (6 PCs).

Fig. 3 illustrates the average classification accuracies achieved by the local PCA models. It can be seen that both local models could provide significantly higher classification results than the global ones. Fig. 3 also shows that the favorable results of the classifier voting and the proposed scheme were obtained by using a few number of selected PCs per local PCA model which can be explained 

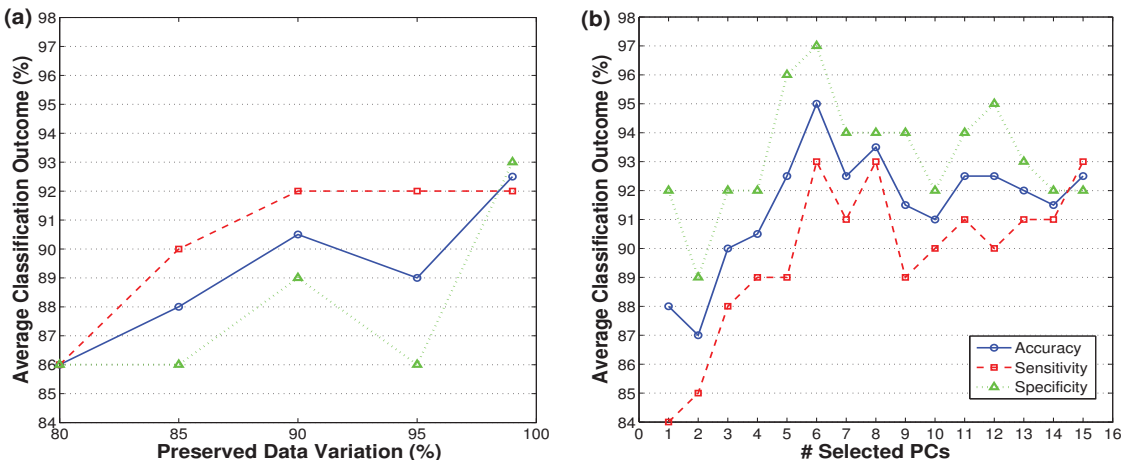

Fig. 2. Average classification outcomes (\%) with the global PCA model. (a) Training the classifier with a subset of the first PCs or (b) with a subset of the selected PCs.
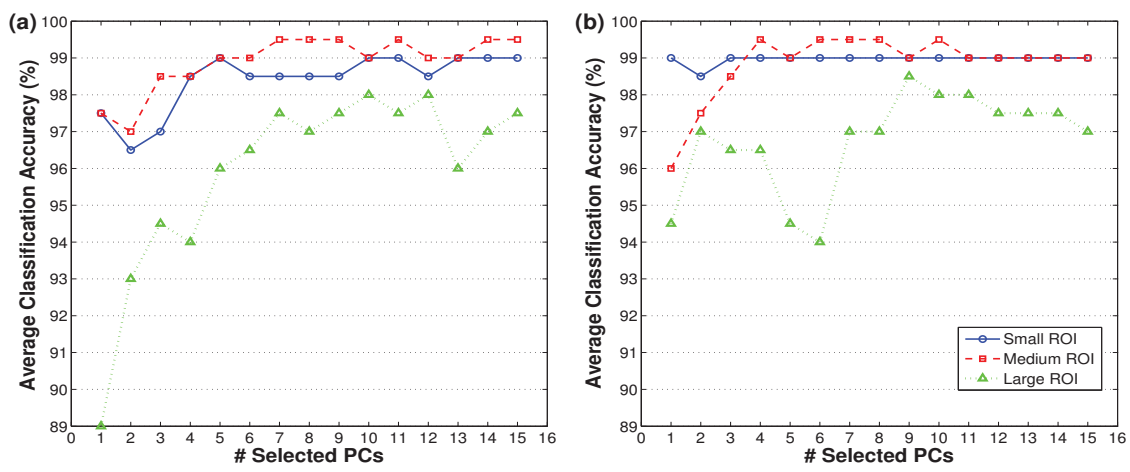

Fig. 3. Average classification accuracies (\%) obtained with the selected PCs of the local PCA models and utilizing (a) the classifier voting scheme and (b) the proposed classification framework.

Table 1. Best average classification accuracies (\%) and their corresponding sensitivity and specificity values obtained with the global PCA model (typed in bold). The (min,max) ranges of the obtained outcomes are also presented.

\begin{tabular}{lll}
\hline Accuracy & Sensitivity & Specificity \\
\hline
\end{tabular}

First PCs

Selected PCs

$\mathbf{9 2 . 5}(80,100) \mathbf{9 2}(80,100) \mathbf{9 3}(80,100)$

$\mathbf{9 5}(80,100) \mathbf{9 3}(80,100) \mathbf{9 7}(80,100)$ 
Table 2. Best average classification accuracies (\%) and their corresponding sensitivity and specificity values obtained with the local PCA models (typed in bold). The $(\min , \max )$ ranges of the obtained outcomes are also presented.

\begin{tabular}{|c|c|c|c|}
\hline & Accuracy & Sensitivity & Specificity \\
\hline \multicolumn{4}{|c|}{ Classifier Voting Scheme } \\
\hline Small ROI & $99(95,100)$ & $\mathbf{9 8}(90,100)$ & $100(100,100)$ \\
\hline Medium ROI & $\mathbf{9 9 . 5}(95,100)$ & $99(90,100)$ & $100(100,100)$ \\
\hline Large ROI & $\mathbf{9 8}(90,100)$ & $\mathbf{9 8}(90,100)$ & $98(90,100)$ \\
\hline \multicolumn{4}{|c|}{ The proposed Scheme } \\
\hline Small ROI & $99(95,100)$ & $98(90,100)$ & $100(100,100)$ \\
\hline Medium ROI & $\mathbf{9 9 . 5}(95,100)$ & $99(90,100)$ & $100(100,100)$ \\
\hline Large ROI & $\mathbf{9 8 . 5}(95,100)$ & $97(90,100)$ & $100(100,100)$ \\
\hline
\end{tabular}

by the following reasons: 1) local regions have less modes of variation than the whole LV and their statistical modeling needs less number of components as well, 2) as shown in Fig. 2(b), selected PCs could provide considerable discriminatory information for the local classification systems.

The best classification results of the global and local PCA models are listed in Tables 1 and 2, respectively.

\section{Discussion}

\subsection{Global versus Local Statistical Modeling}

It has been demonstrated that by using a limited number of the landmark points, a local framework is able to provide better statistical description of LV shapes than its global counterpart. The performances of the local structures, however, depend on the ROI size and tuning this parameter needs a proper compromise between the statistical significance and number of the landmark points. Although the performances of the local classification schemes in detecting myocardial infarction are comparable, direct usage of the local models' parameters and considering the spatial relation between the LV segments would enable the proposed scheme to properly deal with different abnormalities that affect small regions of the myocardium.

\subsection{Feature Selection Utility}

The obtained results confirm the suitability of the feature selection strategy where training a classifier with a small group of the selected PCs could significantly enhance the performance of the same classifier that was trained with a much larger subset of the first PCs. The first five selected modes of variations of 


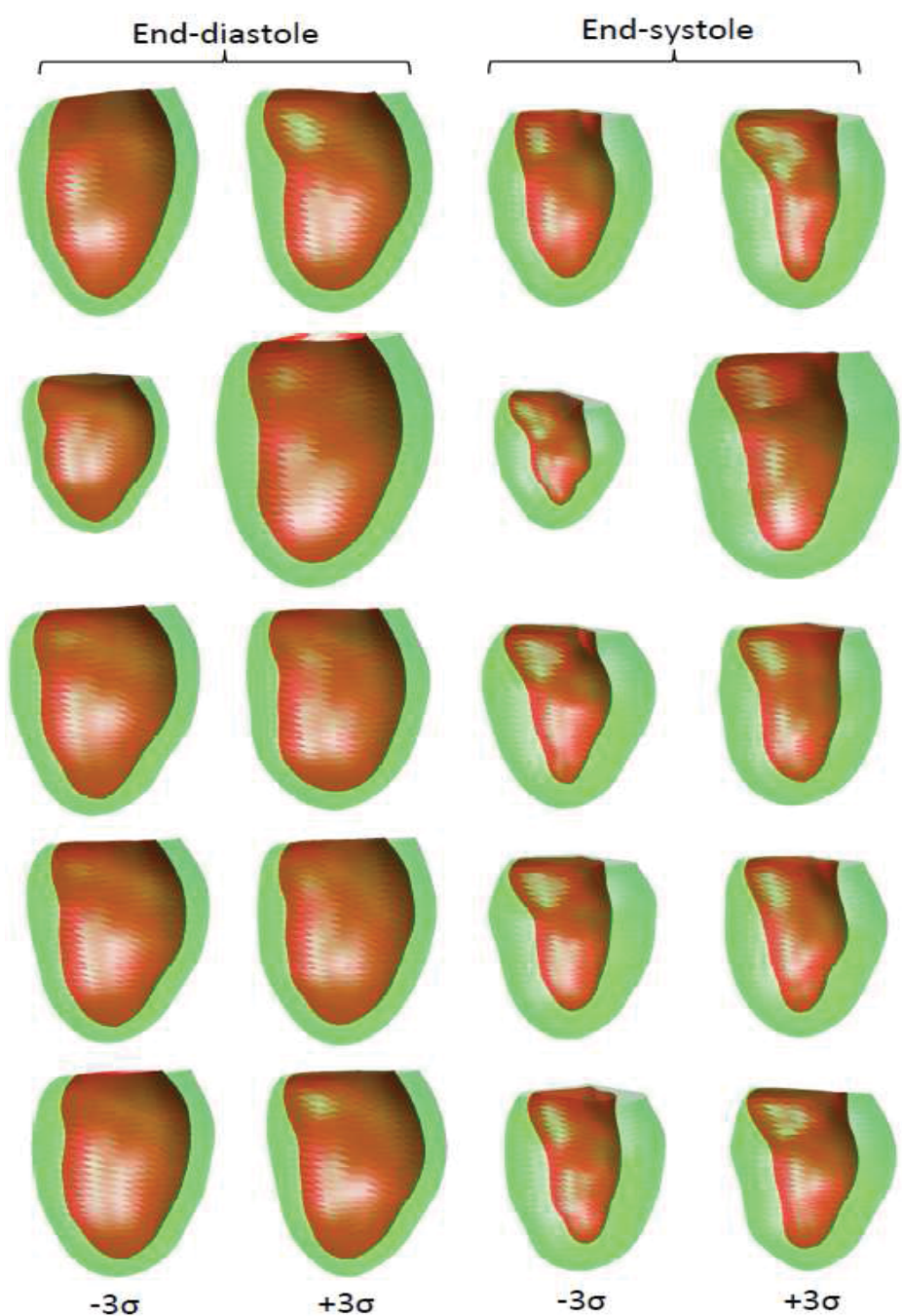

Fig. 4. Variations of the first five selected PCs of the global PCA model at end-diastole and end-systole. From top, PCs 18, 1, 20, 42 and 15 are the selected modes of variation.

the global PCA model are visualized in Fig. 4 to give insight into their characteristics that enable a classifier to discriminate between the normal and infarcted LV shapes. Note that the reason for visualizing the global PCs is that they are easier to interpret than the local PCs.

The left- and righ-hand sides of each LV mode in Fig. 4 correspond to the anteroseptal and inferolateral walls, respectively. The first selected mode (PC 18) describes variations in the curvature of the anterior wall. This PC might have been selected also due to possible difference in contouring left ventricular 
outflow tract (LVOT) in the two studies. The second selected mode (PC 1) explains variations in the LV size. Blunting of the apex and variation of the inferior wall curvature is described by the third selected mode (PC 20). The forth selected mode (PC 42) is associated with the end-systolic variations in the curvatures of the inferior region and the anterior wall. Finally, the fifth selected mode (PC 15) captures the rightward shifting of the apex and variations in the inferior region.

The above-mentioned patho-physiological interpretations are mostly based on the evidences presented in $[11,4]$ and are associated with the process of the LV remodeling due to anterior myocardial infarction. Although the DETERMINE study involves patients with different types of the myocardial infarction, it is wellknown that coronary artery disease occurs most commonly in the left anterior descending (LAD) coronary artery. Therefore, interpretation of the selected PCs based on the findings of $[11,4]$ might be valid for the majority of the subjects in this study.

\section{Conclusion}

A statistical framework has been established based on local PCA models to characterize major modes of LV shape variations. Although local statistical modeling could bring favorable advantages over global parametrization, the adopted strategy for associating the local models' parameters plays a key role in obtaining an efficient local-to-global shape characterization. We hypothesized that the concatenation of the local models' parameters would lead to such efficient characterization. Parameters of each local model were selected based on their significance in discriminating normal and infarcted shapes. Classification outcomes confirmed the superiority of the proposed statistical framework over the global model. They also approved the suitability of the feature selection strategy where utilizing a few number of selected PCs could yield high classification results.

\section{References}

1. Bild, D. E., Bluemke, D. A., Burke, G. L., Detrano, R, Roux, A. V. D., et al. Multiethnic study of atherosclerosis: objectives and design. American Journal of Epidemiology, 156, pp. 871-881, 2002.

2. Cootes, T. F., Cooper, D., Taylor, C. J., Graham, J.: Active shape models-Their training and application. Comput. Vis. Image Understand., vol. 61(1), pp. 38-59, 1995.

3. Cristianini, N., Shawe-Taylore, J.: An Introduction to Support Vector Machines. Cambridge University Press, Cambridge (2000).

4. Di Donato, M., Dabic, P., Castelvecchio, S., Santambrogio, C., Brankovic, J., Collarini, L., et al.: Left ventricular geometry in normal and post-anterior myocardial infarction patients: sphericity index and new conicity index comparisons. Eur. J. Cardio-Thorac. Surg. 29, S225-S230, 2006. 
5. Faghih Roohi, S, Aghaeizadeh Zoroofi, R,: 4D statistical shape modeling of the left ventricle in cardiac MR images. Int J CARS, 8, pp. 335-351, 2013.

6. Fonseca, C. G., Backhaus, M., Bluemke, D. A., Britten, R. D., Do Chung, J., Cowan, B. R., Dinov, I. D., Fin, J. P., Hunter, P. J., Kadish, A. H., Lee, D. C., Lima, J., A., Medrano-Gracia, P., Shivkumar, K., Suinesiaputra, A., Tao, W., Young, A. A. The Cardiac Atlas Project - an imaging database for computational modeling and statistical atlases of the heart. Bioinformatics, 27(16), pp. 2288-2295, 2011.

7. Frangi, A. F., Rueckert, D., Schnabel, J. A., Niessen, W. J.: Automatic construction of multiple-object three-dimensional statistical shape models: application to cardiac modeling. IEEE Trans. Medical Imaging, 21 (9) pp. 1151-1164, 2002.

8. Inza, I., Larranaga, P., Blanco, R., Cerrolaza, A. J., Filter versus wrapper gene selection approaches in DNA microarray domains. Artificial Intelligence in Medicine, 31, pp. 91-103, 2004

9. Jolliffe, I. T.: Principal Component Analysis. NewYork, Springer, 1986.

10. Kadish, A. H., Bello, D., Finn, J., Bonow, R. O., Schaechter, A., Subacius, H., Albert, C., Daubert, J. P., Fonseca, C. G., Goldberger, J. J.: Rationale and design for the defibrillators to reduce risk by magnetic resonance imaging evaluation (DETERMINE) trial. Journal of cardiovascular electrophysiology, 20, pp. 982-987, 2009.

11. Mitchell, G. F., Lamas, G. A., Vaughan, D. E., Pfeffer, M. A.: Left ventricular remodeling in the year after first anterior myocardial infarction: a quantitative analysis of contractile segment lengths and ventricular shape. J Am Coll Cardiol., 19(6), pp. 1136-44, 1992.

12. Perperidis, D., Mohiaddin, R., Rueckert, D.: Construction of a 4D statistical atlas of the cardiac anatomy and its use in classification. In: Proceedings of MICCAI, LNCS 3750, pp. 402-405

13. Rohlf, F., Slice, D.: Extensions of the procrustus method for the optimal superimposition of landmarks. Syst Zool, 39, pp. 40-59, 1990.

14. Stegmann, M. B., Stojstrand, K., Larsen, R.: Sparse modeling of landmark and texture variability using the orthomax criterion. In Int. Symp. Med. Imag., vol. 6144, pp. 485-496, 2006.

15. Suinesiaputra, A., Frangi, A. F., Kaandorp, T. A. M., Lamb, H. J., Bax, J. J., Reiber, J. H. C., Lelieveldt, B. P. F.: Automated detection of regional wall motion abnormalities based on a statistical model applied to multislice short-axis cardiac MR images. IEEE Trans. Medical Imaging, 28(4), pp: 595-607, 2009.

16. Tenenbaum, J. B., De Silva, V., Langford, J. C.: A global geometric framework for nonlinear dimensionality reduction. Science, vol. 290, pp. 2319-2323, 2000.

17. Ye, D. H., Desjardins, B., Hamm, J., Litt, H., Pohl, K. M.: Regional Manifold Learning for Disease Classification. IEEE Trans. Medical Imaging, 33 (6), 2014.

18. Zhang, X., Cowan, B. R., Bluemke, D. A., Finn, J. P., Fonseca, C. G., Kadish, A. H., Lee, D. C., Lima, J. A. C., Suinesiaputra, A., Young, A. A., Medrano-Gracia, P.: Atlas-Based Quantification of Cardiac Remodeling Due to Myocardial Infarction. PLOS ONE, vol. 9 (10), 2014. 